\title{
Modeling the Inherent Damping of High-Power Inverters
}

\author{
Joseba Erdocia, Andoni Urtasun, and Luis Marroyo \\ Department of Electrical, Electronic and Communication Engineering \\ Public University of Navarre (UPNA) \\ Pamplona, Spain \\ ioseba.erdocia@unavarra.es
}

\begin{abstract}
In order to decide whether passive or active damping is required in a three-phase inverter, a previous step is to assess the intrinsic damping of the system. However, few works focus on modeling this damping for DC/AC operation. This paper proposes two models to reproduce the damping sources: the simulation model, used to validate the system in large-signal, and the small-signal model, which can be employed for the controller design. Both models have been validated by means of experimental results for a 1.64 MVA inverter.
\end{abstract}

Keywords-High-power inverter, inherent damping, LC-filter resonance, switching delays

\section{INTRODUCTION}

Three-phase inverters usually include LC-filters at its output instead of L-filters to reduce the global cost and size of the converter. However, LC-filters present the disadvantage of having a resonance inherent to its operation. Although this is not an issue for low-power inverters, in which the resonance is naturally damped, the current tendency to increase the inverter rated power, in order to reduce its cost, is bringing a damping reduction. A too low damping can cause the appearance of resonance-related problems such as oscillations, instability or reduction of the control bandwidth. In consequence, the intrinsic damping of the system should be accurately estimated before considering whether the addition of passive or active damping is required.

In a real inverter, the sources of damping are mainly three: the losses in the inductor, which increases with the frequency [1],[2], the conduction losses of the switches [3], and a lossless phenomenon related to the deadtime and turn-on and turn-off switching delays. The last effect involves an error at the output voltage that has been analyzed in detail in the literature [4]-[6]. Nevertheless, although these articles obtain the voltage error, its effect on the damping of the system is not considered. In [7], the proposed small-signal model does take into account this effect, but it is obtained for a converter working under DC/DC operation. Previous works are mainly focused on estimating the losses or understanding the influence of the deadtime on the output voltage error, instead of obtaining a dynamic model of the power stage which considers the contribution of each effect to the intrinsic damping under $\mathrm{DC} / \mathrm{AC}$ operation.

This article proposes a method that permits modeling accurately the inherent damping of a three-phase inverter. This method is a useful tool since it makes it possible to determine whether the resonance needs to be passively or actively damped, and then to reliably design the control scheme and the controller parameters.

This work was supported by the Spanish State Research Agency (AEI) under grants PID2019-110956RB-I00 /AEI/ 10.13039 and DPI-2016-80641-R, and by the Public University of Navarre through a doctoral scholarship.
The paper is organized as follows. In Section 2, the three main sources of intrinsic damping in an inverter are analyzed. Then, the proposed method for accurately modeling the system damping, which leads to the simulation and small-signal models, is presented in Section 3. The dynamic response of the resulting models is experimentally validated in Section 4 by means of the results obtained with a 1.64 MVA three-phase inverter. Finally, the conclusions are drawn in Section 5.

\section{SOURCES OF DAMPING IN A SWITCHING CELL}

Fig. 1 shows the three-phase inverter with LC-filter, where the main parameters of the system under study are presented in Table I. The resistance $R_{i n v}$ includes the effect of the three sources of damping which exist in the inverter, namely the inductor and semiconductor losses, and the damping related to the switching delays. In relation to the inductor, the resistance $R_{L, 50 \mathrm{~Hz}}$ models its losses at $50 \mathrm{~Hz}$, which are mainly due to the joule effect in the windings. The series resistance of the capacitor is not considered since its effect on the total damping is negligible. As the nominal current is very high, the inverter includes six switching cells per phase and an equal sharing of the current is assumed.

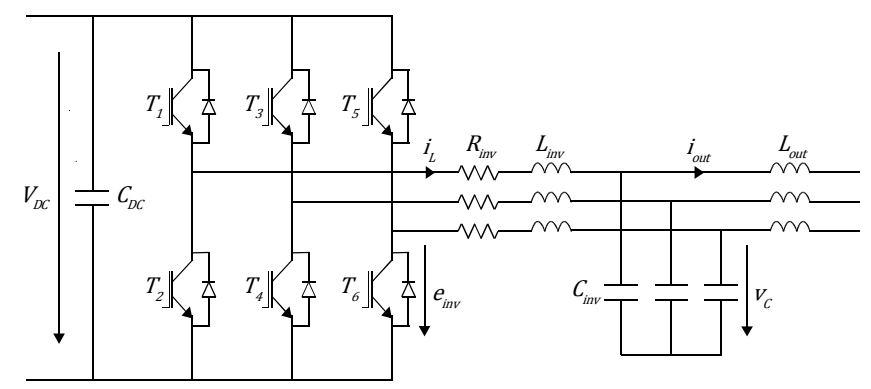

Fig. 1. Three phase inverter with LC filter.

TABLE I. PARAMETERS OF THE SYSTEM

\begin{tabular}{|c|c|}
\hline Parameter & Value \\
\hline \hline DC voltage, $V_{D C}$ & $1200 \mathrm{~V}$ \\
\hline Inverter nominal voltage, $V_{\text {nom }}$ & $630 \mathrm{~V}$ \\
\hline Inverter nominal current, $I_{n o m}$ & $1500 \mathrm{~A}$ \\
\hline Inverter inductance, $L_{i n v}$ & $92 \mu \mathrm{H}$ \\
\hline $50 \mathrm{~Hz}$ resistance of the inductor, $R_{L, 50 \mathrm{~Hz}}$ & $1 \mathrm{m \Omega}$ \\
\hline Inverter capacitance, $C_{i n v}$ & $600 \mu \mathrm{F}$ \\
\hline Resonance frequency, $f_{\text {res }}$ & $677 \mathrm{~Hz}$ \\
\hline Switching frequency, $f_{s w}$ & $3000 \mathrm{~Hz}$ \\
\hline Deadtime, $T_{\text {dead }}$ & $3 \mu \mathrm{s}$ \\
\hline Switching cells per phase & 6 \\
\hline
\end{tabular}


The admittance $Y_{i n v}(s)$, defined as the relation between the inverter voltage $e_{i n v}$ and the inductor current $i_{L}$, can be expressed as

$$
\begin{aligned}
& Y_{\text {inv }}(s)=\frac{i_{L}(s)}{e_{\text {inv }}(s)} \approx \frac{1}{\left(L_{\text {inv }}+L_{\text {out }}\right) \cdot s+R_{\text {inv }}} \cdot \frac{\frac{s^{2}}{\omega_{\text {antires }}^{2}}+1}{\frac{s^{2}}{\omega_{\text {res }}^{2}}+\frac{2 \cdot \xi_{\text {res }}}{\omega_{\text {res }}} \cdot s+1}, \\
& \omega_{\text {res }}=\sqrt{\frac{L_{\text {inv }}+L_{\text {out }}}{L_{\text {inv }} \cdot L_{\text {out }} \cdot C_{f}}}, \\
& \xi_{\text {res }}=\frac{R_{\text {inv }}}{2} \cdot \sqrt{\frac{L_{\text {out }}^{3} \cdot C_{f}}{L_{\text {inv }} \cdot\left(L_{\text {out }}+L_{\text {inv }}\right)^{3}}}, \\
& \omega_{\text {antires }}=\sqrt{\frac{1}{L_{\text {out }} \cdot C_{f}}},
\end{aligned}
$$

where $\omega_{\text {res }}$ is the resonance frequency, $\xi_{r e s}$ is the damping factor of the resonance and $\omega_{\text {antires }}$ is the antiresonance frequency. The approximation shown in (1) and the calculation of (3) are derived in the appendix.

At the resonance frequency, $Y_{i n v}\left(j \cdot \omega_{r e s}\right)$ is inversely proportional to $\xi_{\text {res }}$ and can thus reach very high values, which can cause problems such as oscillations, instability or reduction of the control bandwitdth. For this reason, the damping of the inverter needs to be accurately estimated in order to predict the impact of the resonance on the system.

\section{A. Inductor Losses}

The losses in an inductor are mainly due to two different effects: the joule losses in the copper windings and the magnetic losses in the inductor core. Regarding the former, the conductor resistance can be considered constant as the skin effect can be neglected for the frequencies under study. Nevertheless, this assumption cannot be done for the magnetic losses due to their remarkable dependence on the frequency.

The magnetic core losses could be split in hysteresis loss, eddy current loss and residual loss, though usually the way to calculate them is altogether. In fact, the most extended equation to estimate the average magnetic losses is

$$
p_{V}=k \cdot f^{\alpha} \cdot \widehat{B}_{p}^{\beta},
$$

known as Steinmetz Equation [1], where $p_{V}$ are the power losses per unit volume, $f$ the remagnetization frequency and $\widehat{B}_{p}$ the induction peak. The parameters $k, \alpha$ and $\beta$ are three empirical parameters which are given in the inductor datasheet. Considering that the parameter $\alpha$ is always flowing higher than 1, (5) shows the strong dependence of the magnetic losses on the frequency of the current components through the inductor.

In order to model this dependence, [8] proposes a set of RL impedances which reproduce the resistance and inductance variation with the frequency. Accordingly, the inductor model shown in Fig. 2 is employed so as to consider this correlation. As shown in the figure, the parameters $L_{l f}$, $L_{h f}, R_{l f}$ and $R_{h f}$ have been defined in a way that the inductor model replicates the inductor behavior at $50 \mathrm{~Hz}, R_{L, 50 \mathrm{~Hz}}$ and $L_{50 \mathrm{~Hz}}$, as well as at the resonance frequency, $R_{L, f r e s}$ and $L_{i n v}$. On the basis of this model, Figure 3 (a) and (b) show the change of the resistance $R_{L}$ and the inductance $L$ as a function of frequency.

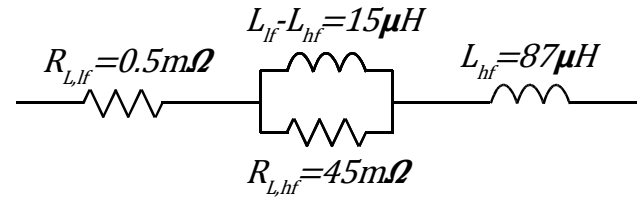

At $50 \mathrm{~Hz}$

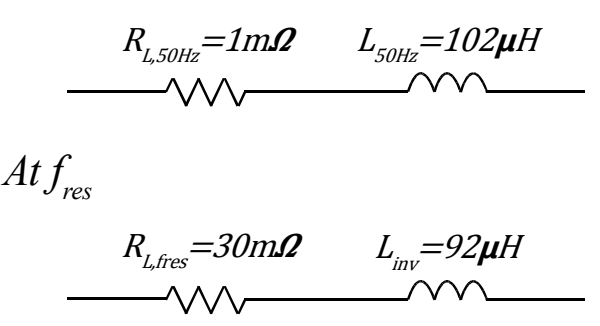

Fig. 2. Inductor impedance model [8].

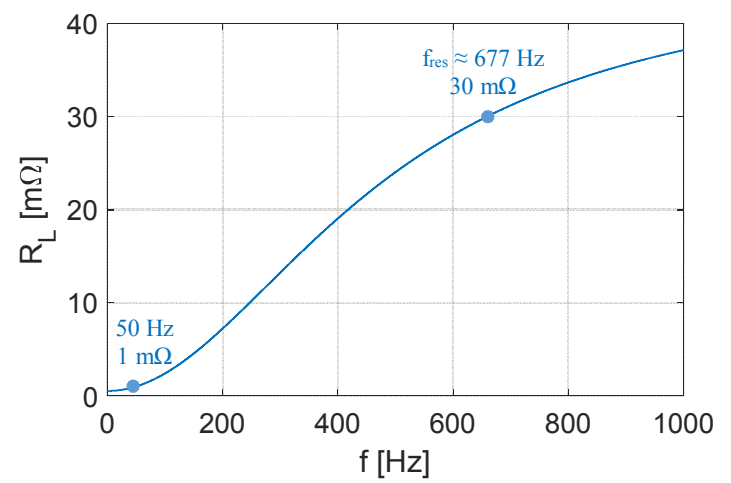

(a)

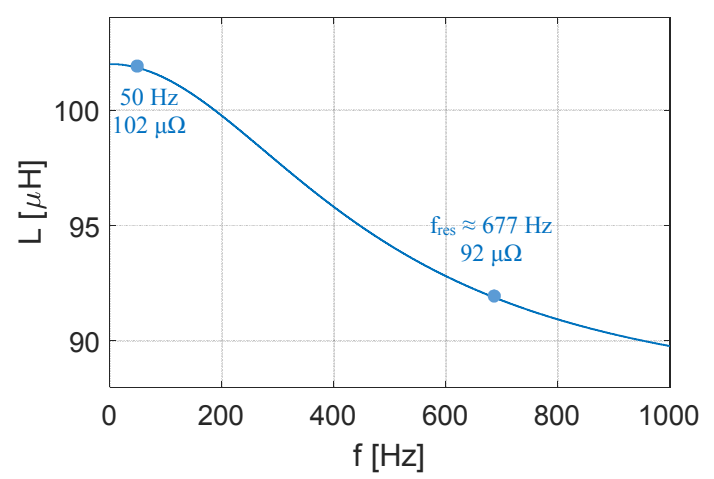

(b)

Fig. 3. Inductor impedance behavior (a) $R_{L}-f$ (b) $L-f$.

\section{B. Switches Conduction Losses}

Figure 4 shows the IGBTs and diodes curves relating the voltage drop $V_{C E}$ and the collector current $I_{C}$, as given in the manufacturer datasheet for a determined junction temperature $T_{j}$ equal to $150^{\circ} \mathrm{C}$. These losses are often modelled by a constant voltage source $U_{F}$ in series with a constant resistance $r_{\text {cond }}$ when the switch is on. From the thermal design viewpoint, this approach is acceptable to estimate the conduction losses at the nominal operating point. However, with respect to the damping contribution, this method is only valid for high currents, where the relation between $V_{C E}$ and $I_{C}$ is linear, as Fig. 4 shows.

In order to analyze the real contribution to the damping, Fig. 5 shows the variation of the small-signal resistance $r_{\text {cond }}$ 
according to the current $I_{C}$. As can be observed, $r_{\text {cond }}$ changes completely with the operating point, providing a much important influence over the intrinsic damping for low currents. In both cases, IGBT and diode, the resistances are higher than $20 \mathrm{~m} \Omega$ when the current $I_{C}$ tends to 0 but remain almost constant to $5 \mathrm{~m} \Omega$ when the current $I_{C}$ is higher than $100 \mathrm{~A}$.

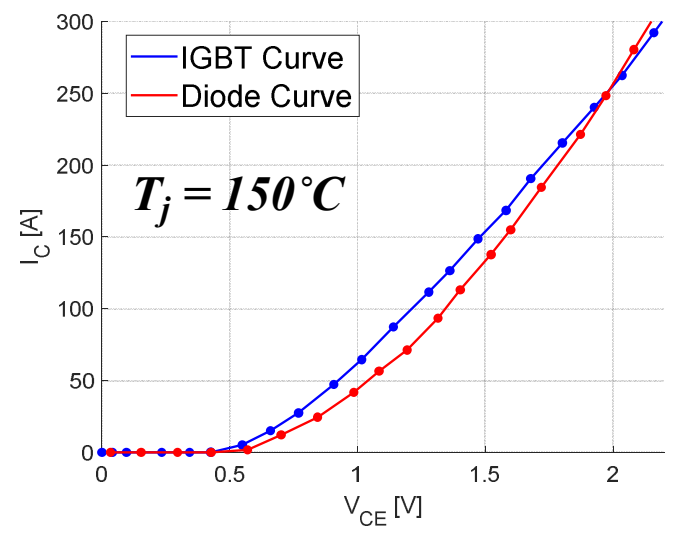

Fig. 4. $I_{C}-V_{C E}$ characteristic curves of the IGBT and diode.

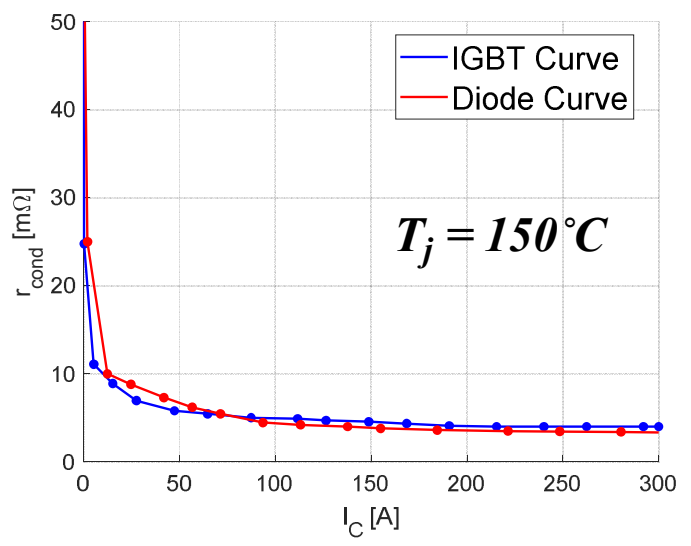

Fig. 5. $\mathrm{r}_{\text {cond }}-\mathrm{I}_{\mathrm{C}}$ characteristic curves of the IGBT and diode.

\section{Switching Delays}

The deadtime, $T_{\text {dead }}$, and the IGBTs turn-on and turn-off delays, $t_{d, o n}$ and $t_{d, \text { off }}$, cause a drop on the inverter output voltage whose value depends on the level of current conducted by the switches. Thus, this phenomenon behaves as a nonlinear resistance that contributes to the damping of the system.
Fig. 6 shows the switching time delay $T_{d}$ of the utilized semiconductors, for different $T_{\text {dead }}$ and considering the data given by the switches manufacturer. As can be observed, $T_{d}$ depends considerably on the value and sign of $i_{s w}$ :

- For $i_{s w}>0$ (right side of the figure), the IGBT is conducting so $T_{d}$ will be equal to the IGBT turn-off delay, $t_{d, o f f}$.

- For $i_{s w}<0$ (left side of the figure), the diode is conducting and it will continue switched on until the complementary IGBT of the switching cell turns on, so $T_{d}$ will be equal to the deadtime plus the IGBT turnon delay, $T_{\text {dead }}+t_{d, \text { on }}[5]$.

For the subsequent analysis, a deadtime equal to $3 \mu$ s has been selected in order to ensure a reliable operation of the inverter as well as to guarantee an extended lifetime of the system components.

In a switching period, the upper and lower semiconductors of the cell switch with different currents, $i_{s w, u p}$ and $i_{s w, l o w}$, respectively, that can be expressed as

$$
\begin{gathered}
i_{s w, u p}=\left\langle i_{s w}>_{T_{s w}}+\frac{\Delta i_{s w}}{2},\right. \\
i_{s w, \text { low }}=-\left(<i_{s w}>_{T_{s w}}-\frac{\Delta i_{s w}}{2}\right),
\end{gathered}
$$

where $\left\langle i_{s w}\right\rangle_{T_{s w}}$ is the average current of the switching cell and $\Delta i_{s w}$ is the switching current ripple. As a results of this, the switching time delays $T_{d, u p}$ and $T_{d, l o w}$ are not equal and this difference causes a drop at the output voltage per switching period [7],

$$
<v_{d r o p}>_{T_{s w}}=\frac{V_{D C}}{2} \cdot \frac{T_{d, l o w}-T_{d, u p}}{T_{s w}} .
$$

Fig. 7 depicts the voltage drop $\left\langle v_{d r o p}\right\rangle_{T_{s w}}$ as a function of the average current $\left\langle i_{s w}\right\rangle_{T_{s w}}$, considering the time delays shown in Fig. 6 for $T_{\text {dead }}=3 \mu \mathrm{s}$, and for different current ripples $\Delta i_{s w}$. As can be observed, slight variations of $\left\langle i_{s w}\right\rangle_{T_{s w}}$ cause a considerable change of the voltage drop for low currents, but the derivate of this voltage drop is reduced with the increase of $\left\langle i_{s w}\right\rangle_{T_{s w}}$. A Similar effect is produced for negative values of the current $\left\langle i_{s w}\right\rangle_{T_{S w}}$ due to symmetry.
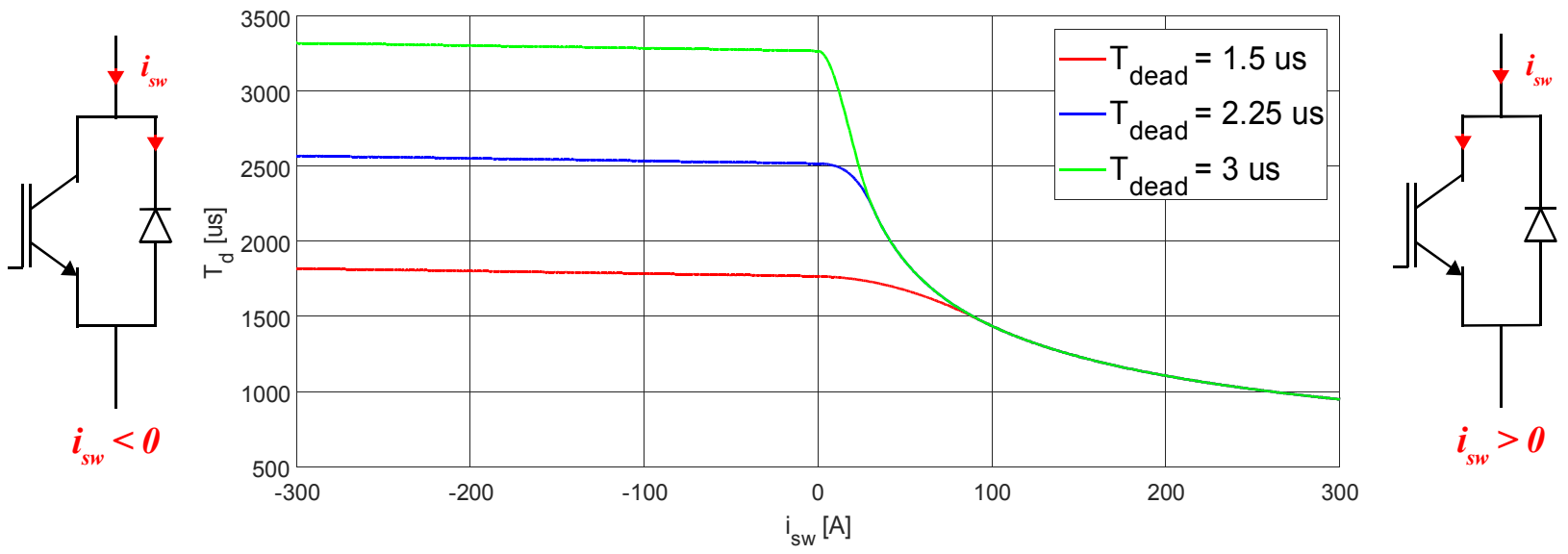

Fig. 6. Time delay $T_{d}$ as a function of the current $i_{s w}$ for different $T_{\text {dead }}$. 
According to Fig. 7, this phenomenon can be modelled as a non-linear resistance $r_{T_{d}}$ highly dependent on the switching cell current $\left\langle i_{s w}\right\rangle_{T_{s w}}$ and Fig. 8 shows this correlation for different current ripples $\Delta i_{s w}$. As stated in Fig. 8, the resistance $r_{T_{d}}$ presents considerably high values for low currents that increase the effective damping of the inverter [7]. Nevertheless, high values of $\left\langle i_{s w}\right\rangle_{T_{s w}}$ cause the resistance $r_{T_{d}}$ to drop, thus reducing its contribution to the damping. It must be noted that, for very low currents, the resistance estimation loses accuracy due to the problems mentioned in [5].

This phenomenon does not cause losses in the inverter since the energy absorbed by the resistance $r_{T d}$ is transferred back to the capacitor of the dc bus instead of being converted to heat [7], so the inverter's efficiency is not reduced.

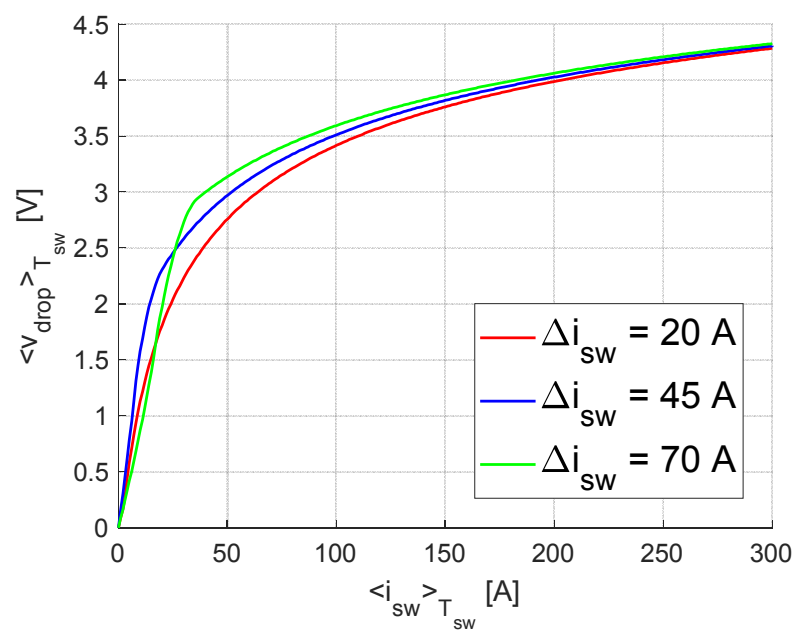

Fig. 7. Output voltage error due to switching delays.

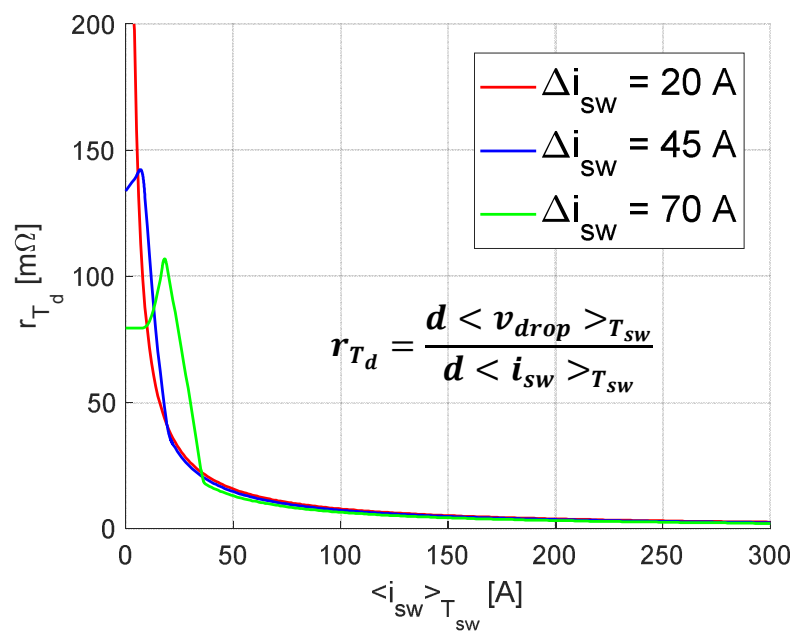

Fig. 8. Non-linear resistance $r_{T d}$ due to switching delays.

\section{MODELING OF THE INVERTER DAMPING}

In order to model the three main sources of damping in an inverter, presented in Section 2, two cases are considered: the simulation model, which is used to validate the system in large-signal, and the small-signal model, employed to design the control parameters, usually carried out for the worst operating point.

Regarding the simulation model, the impedance model proposed in Fig. 2 is considered so as to reproduce the different behaviors of the inductor according to the different current components. In relation to switches conduction losses, the characteristics curves of the IGBTs and diodes can be introduced into the non-ideal semiconductor model available in most simulation softwares. Moreover, the manufacturer usually provides these curves for two different junction temperatures, $25^{\circ} \mathrm{C}$ and $150^{\circ} \mathrm{C}$, which are introduced in the semiconductor model in order to reproduce the influence of the temperature. Nevertheless, generally the switching delays curves cannot be inserted into those non-ideal devices but can be programmed in an external $\mathrm{C}$ block together with the deadtime. The addition of these phenomena in this manner provides the simulation model with the capacity of reproducing the large-signal operation of the inverter.

These sources of damping are also considered in the small-signal model, where the inductor behavior is again modelled as the impedance presented in Fig. 2. In order to model the conduction and switching behavior of the switches, the small-signal resistances shown in Fig. 5 and Fig. 8 are considered. However, those resistances are defined by assuming an operating point with constant current, while in $\mathrm{DC} / \mathrm{AC}$ operation the output current will be sinusoidal. For this reason, it is proposed to define two equivalent resistances, $R_{e q, c o n d}$ and $R_{\text {eq,Td }}$, as a function of the RMS phase current $I_{R M S}$. Figure 9 shows the value of these resistances and the switch total resistance, $R_{e q, s w}$, for different values of $I_{R M S}$. These values have been obtained using the simulation model described above and searching for the equivalent resistance, which reproduces the same resonant behavior after applying a step in the modulation signal of the inverter for different levels of current. In this test, an ideal AC current source has been incorporated at the inverter output to avoid providing the system with extra damping. When comparing Fig. 9 with Fig. 5 and Fig. 8, one must keep in mind that the latter figures are defined for one switching cell, while Fig. 9 refers to one inverter phase, consisting of 6 switching cells in parallel.

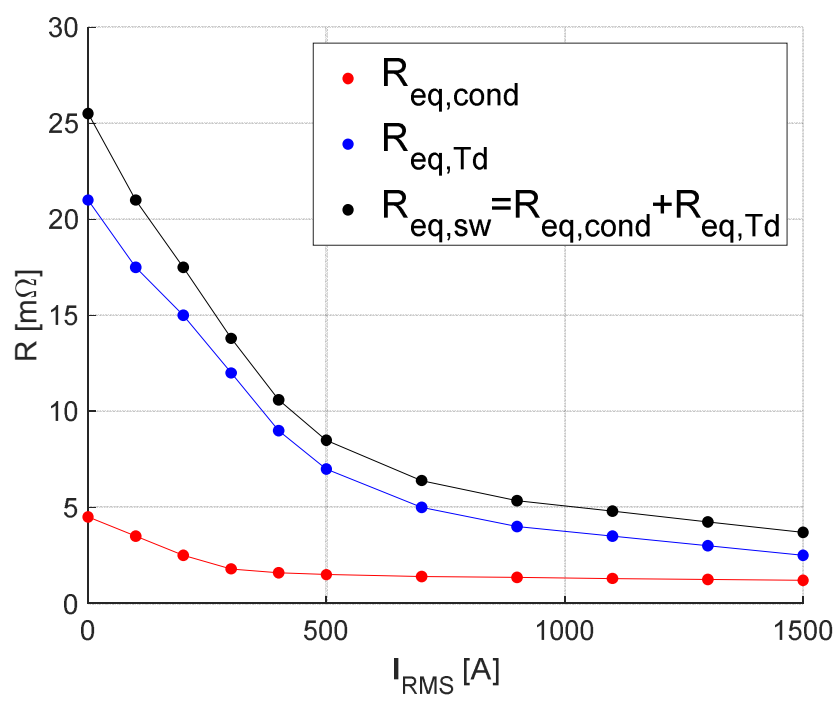

Fig. 9. $R_{e q}-I_{R M S}$ switches curves.

Figure 10 shows the proposed small-signal model for considering the intrinsic damping provided by one phase of the inverter. This model permits estimating the system response at the relevant frequencies, including $50 \mathrm{~Hz}$ and the resonance frequency. Taking everything into account, and considering the parameters shown in Table I, the intrinsic 

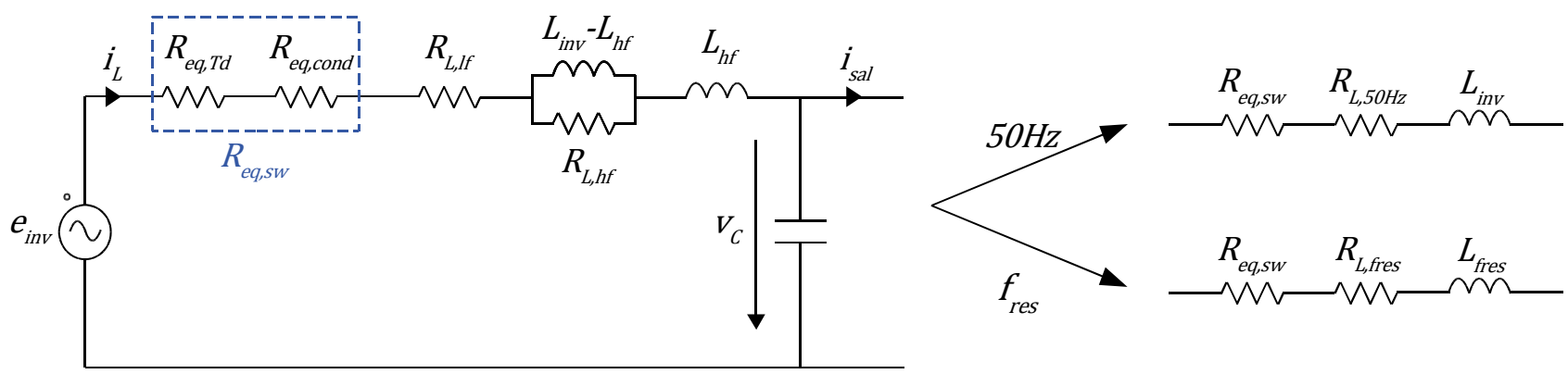

Fig. 10. Small-signal model proposed for including the sources of real damping damping of the system $\xi_{\text {res }}$ can be calculated from (3) as

$$
\xi_{\text {res }}=\frac{R_{\text {eq,cond }}+R_{e q, T_{d}}+R_{L, f_{\text {res }}}}{2} \cdot \sqrt{\frac{L_{\text {out }}^{3} \cdot C_{f}}{L_{\text {inv }} \cdot\left(L_{\text {inv }}+L_{\text {out }}\right)^{3}}} .
$$

According to this, Fig. 11 shows the variation of the damping $\xi_{\text {res }}$ with the phase current $I_{R M S}$ for three different cases: under stand-alone operation connected to a current source (which does not provide extra damping), connected to a weak grid of SCR equal to 1.5 , and connected to a strong grid of SCR equal to 8 . As can be observed, the damping provided by the effects analyzed decreases with the current due to the reduction of the switches resistances.

As a result, the proposed models permit estimating the intrinsic damping of the system at the worst-case scenario in order to reliably design the controller parameters. The worst-case scenario must be defined according to the operation mode of the inverter and the small-signal model must be evaluated for that corresponding point. On the one hand, in a stand-alone system, the load generally increases the damping so the no-load operation represents the worst-case scenario [9]. In this point, the intrinsic damping $\xi_{\text {res }}$ provided by the inverter is maximum as Fig. 11 shows. On the other hand, for a grid-connected system, the grid hardly contributes to the total damping. Consequently, the worst-case scenario is when the inverter exchanges rated current with the grid since the damping provided by the inverter in this operating point is minimum [10], as can be observed in Fig. 11.

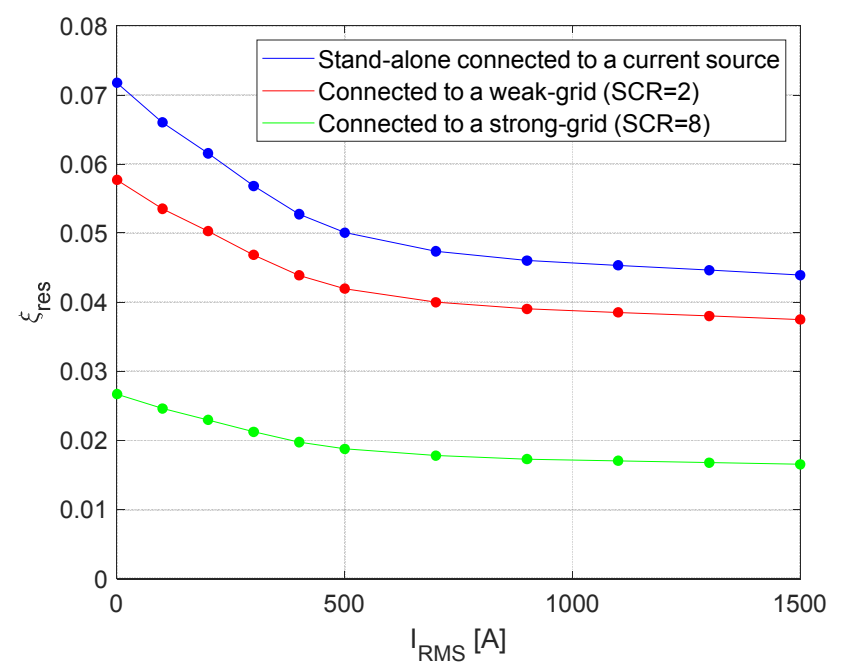

Fig. 11. $\xi_{\text {res }}-I_{R M S}$ under different operation modes

\section{EXPERIMENTAL VALIDATION}

For the validation of the proposed simulation and small-signal models, the three-phase inverter with LC-filter shown in Fig. 12 has been tested. The rated power of the inverter is $1.64 \mathrm{MVA}$ with a rated voltage of $630 \mathrm{~V} / 50 \mathrm{~Hz}$ and a rated current of $1500 \mathrm{~A}$. Other system parameters are listed in Table I.

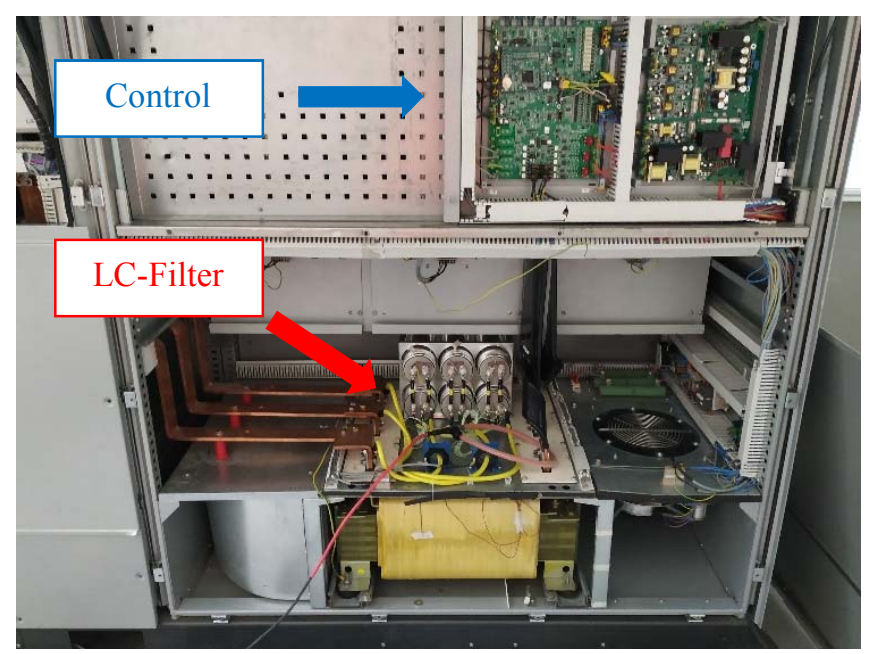

Fig. 12. Experimental 1.64 MVA three-phase inverter

The test is carried out in open-circuit, i.e. without load, operating point in which the system damping is mainly due to the three effects explained in previous sections. In this experiment, the inverter operates with no feedback control and generates sinusoidal voltage $e_{i n v}$ at $50 \mathrm{~Hz}$. Abruptly, the phase-to-phase RMS reference is changed from 260 to $630 \mathrm{~V}$, which causes the appearance of an oscillation at the resonance frequency in the inductor current and capacitor voltage, whose amplitude and duration is directly related to the intrinsic damping of the inverter. Figure 13 shows the variation of the inductor current $i_{L}$ and the line voltage $v_{C}$ caused by the voltage step in the experimental setup, while Fig. 14 (a) and (b) show the variation of $i_{L}$ and $v_{C}$ after carrying out the same test in the simulation and small-signal models, respectively.

As Fig. 13 and Fig. 14 show, the dynamic response of $i_{L}$ and $v_{C}$ obtained in the experimental test is similar to the response obtained with the both models proposed, simulation and small-signal. In the three cases, the oscillation presents the frequency of the LC-filter resonance (around $680 \mathrm{~Hz}$ ) and the intrinsic damping of the inverter at that frequency is properly modelled considering that the amplitude and duration of the oscillations are practically identical. 


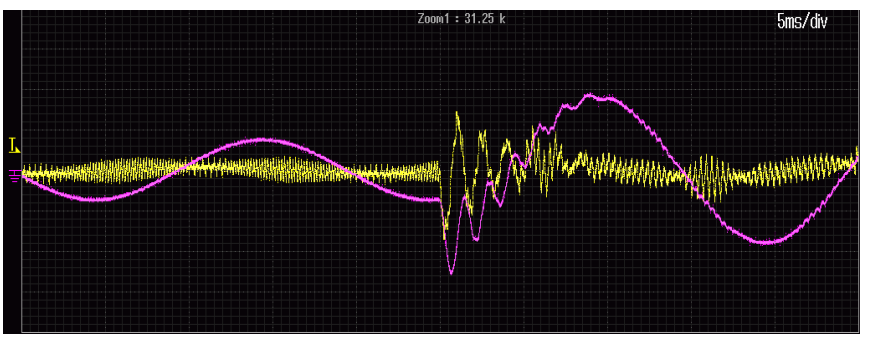

$\mathrm{i}_{\mathrm{Lr}}(500 \mathrm{~A} / \mathrm{div})$

$\mathrm{v}_{\mathrm{Crs}}(500 \mathrm{~V} / \mathrm{div})$

Fig. 13. Phase $r$ inductor current $i_{L r}$ and line output voltage $v_{C r s}$ Experimental results

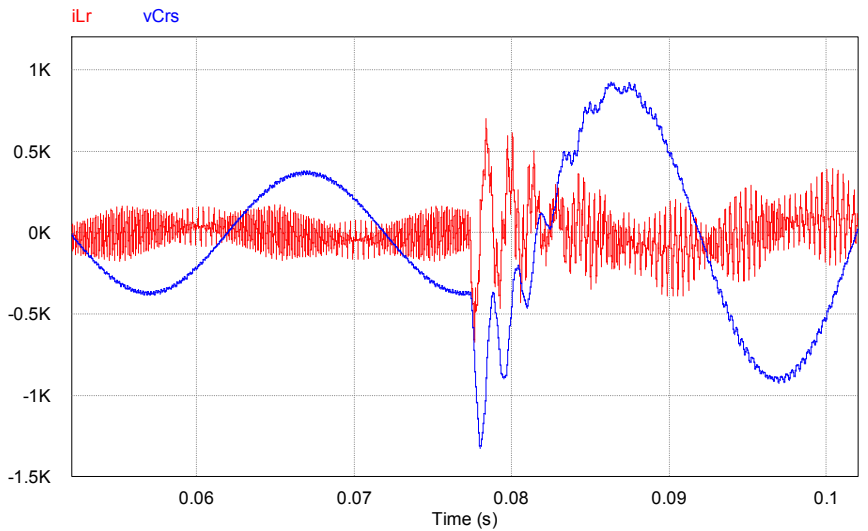

(a)

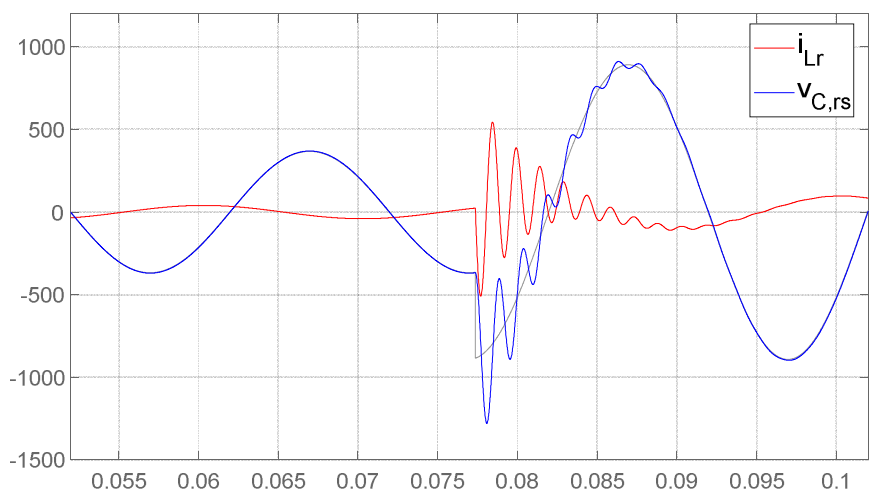

(b)

Fig. 14. Phase $r$ inductor current $i_{L r}$ and line output voltage $v_{C r s}$ (a) Simulation model results (b) Theoretical model results

Although this operating point represents the worst-case scenario in a stand-alone system, a sufficient damping is observed $\left(\xi_{\text {res }}=0.07\right)$, and the resonance-related oscillations last less than $10 \mathrm{~ms}$ not representing a problem. Therefore, it can be concluded that active damping is not required under this operation mode.

\section{CONCLUSIONS}

In order to decide whether passive or active damping is required in a three-phase inverter to avoid problems related to the LC-filter resonance, a previous step is to assess the intrinsic damping of the system. This paper first presents the three main sources of damping in a switching cell: the inductor losses, the switching conduction losses and the switching delays. Then, it proposes two manners of modeling the inverter damping: the simulation model, to be employed for large-signal validation, and the small-signal model, which can be used as an easy and useful tool for the control design. These models have been validated for no-load conditions by means of experimental results obtained for a real 1.64 MVA three-phase inverter.

\section{APPENDIX}

This appendix derives how to obtain the admittance approximation shown in (1) and the calculation of the resonance frequency and damping equations shown in (2) and (3). In order to begin, the admittance equation, without neglecting any term, can be expressed as

$$
\begin{aligned}
& Y_{\text {inv }}(s)=\frac{i_{L}(s)}{e_{\text {inv }}(s)}=\frac{L_{\text {out }} \cdot C_{f} \cdot s^{2}+1}{\operatorname{den}(s)} \\
& \operatorname{den}(s)=a_{3} \cdot s^{3}+a_{2} \cdot s^{2}+a_{1} \cdot s+a_{0}
\end{aligned}
$$

where

$$
\begin{gathered}
a_{3}=L_{\text {inv }} \cdot L_{\text {out }} \cdot C_{f} \\
a_{2}=L_{\text {out }} \cdot C_{f} \cdot R_{\text {inv }} \\
a_{1}=L_{\text {out }}+L_{\text {inv }} \\
a_{0}=R_{\text {inv }}
\end{gathered}
$$

The admittance $Y_{\text {inv }}(s)$ presents a third-order denominator. Thus, there are 3 poles that can be divided into a real pole, related to the low frequency behavior of the circuit and a pair of complex poles related to the resonance. Accordingly, the denominator of the admittance can be approximated as

$$
\operatorname{den}_{a p}(s)=\left[\left(L_{\text {inv }}+L_{\text {out }}\right) \cdot s+R_{\text {inv }}\right] \cdot\left(\frac{s^{2}}{\omega_{\text {res }}^{2}}+\frac{2 \cdot \xi_{\text {res }}}{\omega_{\text {res }}} \cdot s+1\right)
$$

The approximation (A.7) can be also expressed as a thirdorder polynomial

$$
\operatorname{den}_{a p}(s)=b_{3} \cdot s^{3}+b_{2} \cdot s^{2}+b_{1} \cdot s+b_{0}
$$

where

$$
\begin{gathered}
b_{3}=\frac{L_{i n v}+L_{\text {out }}}{\omega_{\text {res }}^{2}} \\
b_{2}=\frac{2 \cdot \xi_{\text {res }}}{\omega_{\text {res }}} \cdot\left(L_{\text {inv }}+L_{\text {out }}\right)+\frac{R_{\text {inv }}}{\omega_{\text {res }}^{2}} \\
b_{1}=\left(L_{\text {inv }}+L_{\text {out }}\right)+\frac{2 \cdot \xi_{\text {res }}}{\omega_{\text {res }}} \cdot R_{\text {inv }} \\
b_{0}=R_{\text {inv }}
\end{gathered}
$$

From the comparison between the polynomial coefficients of (A.2) and (A.8), the value of the two degrees of freedom $\omega_{\text {res }}(2)$ and $\xi_{\text {res }}(3)$ is obtained as follows

$$
\begin{gathered}
a_{0}=b_{0} \\
a_{3}=b_{3} \rightarrow \omega_{\text {res }}=\sqrt{\frac{L_{\text {inv }}+L_{\text {out }}}{L_{\text {inv }} \cdot L_{\text {out }} \cdot C_{f}}, f_{\text {res }}=\frac{\omega_{\text {res }}}{2 \cdot \pi}} \\
a_{2}=b_{2} \rightarrow \xi_{\text {res }}=\frac{R_{\text {inv }}}{2} \cdot \sqrt{\frac{L_{\text {out }}^{3} \cdot C_{f}}{L_{\text {inv }} \cdot\left(L_{\text {inv }}+L_{\text {out }}\right)}}
\end{gathered}
$$



that

However, the definitions made in (A.15) and (A.16) cause

$$
a_{1} \not \approx b_{1}
$$

The difference between both coefficients is calculated by replacing (A.15) and (A.16)

$$
\begin{gathered}
b_{1}-a_{1}=\frac{2 \cdot \xi_{\text {res }}}{\omega_{\text {res }}} \cdot R_{\text {inv }}=\frac{R_{\text {inv }}^{2} \cdot L_{\text {out }}^{2} \cdot C_{f}}{\left(L_{\text {inv }}+L_{\text {out }}\right)^{2}} \\
b_{1}-a_{1} \ll\left(L_{\text {inv }}+L_{\text {out }}\right) \rightarrow a_{1} \approx b_{1}
\end{gathered}
$$

As can be observed, the difference between both coefficients is small respect to (A.5) and can be neglected.

Figure A.1 (a) and (b) show the variation of the frequency and damping, respectively, of the resonance poles for different values of $L_{\text {out }}$. As it is stated, the results obtained from calculating the poles by means of (A.2) and using the equations (A.14) and (A.15) are compared. The parameters calculated with both methods are really similar, and thus the accuracy of (A.14) and (A.15) is validated.

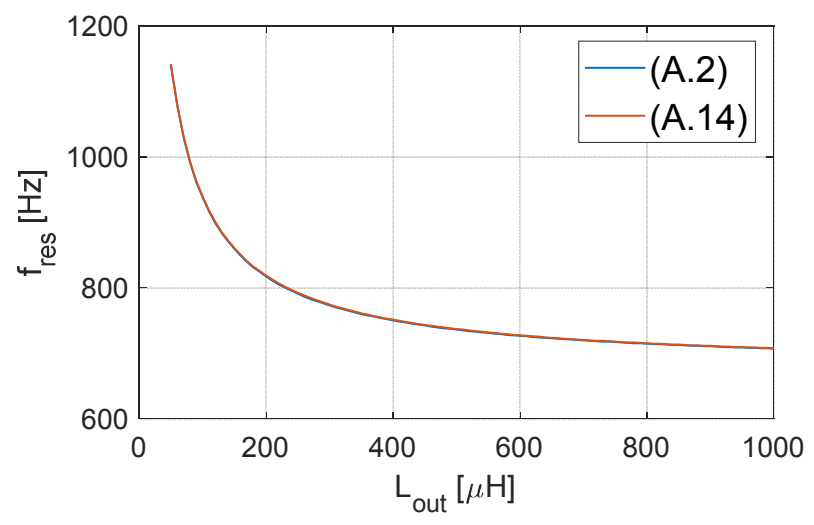

(a)

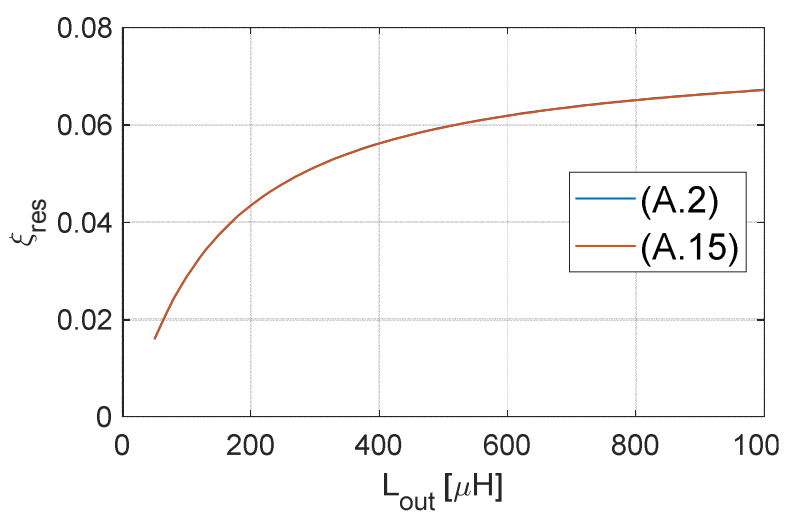

(b)

Fig. A1 Comparison of (A.2) with (A.14) and (A.15) (a) $f_{\text {res }}-L_{o u t}$ (b) $\xi_{\text {res }}-L_{\text {out }}$

\section{ACKNOWLEDGMENT}

The authors gratefully acknowledge the financial and ongoing support of INGETEAM POWER TECHNOLOGY.

\section{REFERENCES}

[1] A. Brockmeyer and L. Schilting, "Modelling of dynamic losses in magnetic material" Fifth Eur. Conf. Power Electron. Appl., pp. 112-117, 1993.

[2] J. Mühlethaler, J. W. Kolar, and A. Ecklebe, "Loss modeling of inductive components employed in power electronic systems," 8 th Int. Conf. Power Electron. - ECCE Asia "Green World with Power Electron. ICPE 2011-ECCE Asia, pp. 945-952, 2011.

[3] U. Drofenik and J. Kolar, "A general scheme for calculating switching-and conduction-losses of power semiconductors in numerical circuit simulations of power electronic systems," Int. power Electron. Conf. IPEC-Niigata 2005, pp. 1-7, 2005.

[4] J.-W. Choi and S.-K. Sul, "Inverter output voltage synthesis using novel dead time compensation," IEEE Trans. Power Electron., vol. 11, no. 2, pp. 221-227, 1996.

[5] G. Shen, W. Yao, B. Chen, K. Wang, K. Lee, and Z. Lu, "Automeasurement of the inverter output voltage delay curve to compensate for inverter nonlinearity in sensorless motor drives," IEEE Trans. Power Electron., vol. 29, no. 10, pp. 5542-5553, 2014.

[6] T. Mannen and H. Fujita, "Dead-time compensation method based on current ripple estimation," IEEE Trans. Power Electron., vol. 30, no. 7, pp. 4016-4024, 2015.

[7] F. Krismer, D. O. Boillat, and J. W. Kolar, "Modeling the Output Impedance of a T-Type Power Converter," IEEE Trans. Power Electron., vol. 31, no. 12, pp. 8052-8057, 2016.

[8] L. Heinemann, R. Schulze, P. Wallmeier, and H. Grotstollen, "Modeling of high frequency inductors," PESC Rec. - IEEE Annu. Power Electron. Spec. Conf., vol. 2, pp. 876-883, 1994.

[9] R. Zamodzki, C. M. O. Stein, E. G. Carati, J. P. Da Costa, and R. Cardoso, "Stability analysis of three-phase stand-alone inverters with LCL filter," 2016 12th IEEE Int. Conf. Ind. Appl. INDUSCON 2016, 2017.

[10] A. Rodriguez-Cabero, M. Prodanovic, and J. Roldan-Perez, "Analysis of dynamic properties of VSCs connected to weak grids including the effects of dead time and time delays," IEEE Trans. Sustain. Energy, vol. 10, no. 3, pp. 1066-1075, 2019. 